\title{
Synthesizing a Contrast-Enhancement Map in Patients with High-Grade Gliomas Based on a Postcontrast MR Imaging Quantification Only
}

\author{
(D) M. Warntjes, ${ }^{(D)}$. Blystad, (D) A. Tisell, and DE.-M. Larsson
}

\begin{abstract}
BACKGROUND AND PURPOSE: Administration of a gadolinium-based contrast agent is an important diagnostic biomarker for bloodbrain barrier damage. In clinical use, detection is based on subjective comparison of native and postgadolinium-based contrast agent T7-weighted images. Quantitative MR imaging studies have suggested a relation between the longitudinal relaxation rate and protondensity in the brain parenchyma, which is disturbed by gadolinium-based contrast agents. This discrepancy can be used to synthesize a contrast-enhancement map based solely on the postgadolinium-based contrast agent acquisition. The aim of this study was to compare synthetic enhancement maps with subtraction maps of native and postgadolinium-based contrast agent images.
\end{abstract}

MATERIALS AND METHODS: For 14 patients with high-grade gliomas, quantitative MR imaging was performed before and after gadolinium-based contrast agent administration. The quantification sequence was multidynamic and multiecho, with a scan time of 6 minutes. The 2 image stacks were coregistered using in-plane transformation. The longitudinal relaxation maps were subtracted and correlated with the synthetic longitudinal relaxation enhancement maps on the basis of the postgadolinium-based contrast agent images only. ROIs were drawn for tumor delineation.

RESULTS: Linear regression of the subtraction and synthetic longitudinal relaxation enhancement maps showed a slope of $1.02 \pm 0.19$ and an intercept of $0.05 \pm 0.12$. The Pearson correlation coefficient was $0.861 \pm 0.059$, and the coefficient of variation was $0.18 \pm 0.04$. On average, a volume of $1.71 \pm 1.28 \mathrm{~mL}$ of low-intensity enhancement was detected in the synthetic enhancement maps outside the borders of the drawn ROI.

CONCLUSIONS: The study shows that there was a good correlation between subtraction longitudinal relaxation enhancement maps and synthetic longitudinal relaxation enhancement maps in patients with high-grade gliomas. The method may improve the sensitivity and objectivity for the detection of gadolinium-based contrast agent enhancement.

ABBREVIATIONS: $d R_{1}=R_{1}$ enhancement; $G B C A$ = gadolinium-based contrast agent; $P D=$ proton-density; $R_{1}=$ longitudinal relaxation rate; $R_{2}=$ transverse relaxation rate

$\mathrm{T}$ he clinical use of gadolinium-based contrast agents (GBCAs) is a diagnostic biomarker for detecting blood-brain barrier damage, a frequent finding in high-grade gliomas. In clinical practice, a native T1-weighted image is acquired to depict the baseline, followed by administration of a gadolinium-based contrast agent. After 5-10 minutes, the T1-weighted acquisition is repeated to show potential leakage of gadolinium-based contrast

Received February 16, 2018; accepted after revision September 24.

From the Centre for Medical Image Science and Visualization (M.W., I.B.., A.T.), Division of Cardiovascular Medicine (M.W.) and Departments of Radiology (I.B.) and Radiation Physics (A.T.), Department of Medical and Health Sciences, Linköping University, Linköping, Sweden; SyntheticMR AB (M.W.), Linköping, Sweden; and Department of Surgical Sciences and Radiology (E.-M.L.), Uppsala University, Uppsala, Sweden.

Please address correspondence to Marcel Warntjes, CMIV, University Hospital Linköping, 58185 Linköping, Sweden; e-mail: marcel.warntjes@cmiv.liu.se

http://dx.doi.org/10.3174/ajnr.A5870 agent into the brain parenchyma, visible as signal enhancement. Typically, the resulting images are shown juxtaposed, and contrast enhancement is estimated by a subjective visual evaluation of the pre- and postcontrast images.

A challenge when using conventional T1-weighted images is that the signal intensity has an arbitrary scale, affected by scanner imperfections such as $B_{1}$ inhomogeneity and coil sensitivity. These prohibit the use of quantitative measures of actual GBCA uptake, which is reported to add value to the assessment and prediction of the outcome of patients with high-grade gliomas. ${ }^{1,2}$ Even though contrast enhancement is an important feature when assessing high-grade gliomas, these tumors are also known to infiltrate into the peritumoral edema. ${ }^{3}$ Tumor infiltration is difficult to detect visually with conventional MR images, and quantitative measurements may therefore add information. 
Table 1: Overview of patient details

\begin{tabular}{lcl}
\hline Patient Sex & Age (yr) & \multicolumn{1}{c}{ WHO 2007 } \\
\hline Male & 68 & Glioblastoma IV \\
Female & 57 & Glioblastoma IV \\
Male & 63 & Anaplastic oligodendroglioma III \\
Female & 58 & Glioblastoma \\
Male & 69 & Glioblastoma \\
Male & 71 & Glioblastoma \\
Male & 65 & Gliosarcoma \\
Female & 65 & Anaplastic oligodendroglioma \\
Female & 45 & Glioblastoma \\
Male & 65 & Glioblastoma \\
Male & 79 & Glioblastoma \\
Male & 45 & Glioblastoma \\
Male & 72 & Glioblastoma \\
Male & 74 & Glioblastoma \\
\hline
\end{tabular}

Note:-WHO indicates World Health Organization.

Recent developments in MR imaging quantification of the longitudinal relaxation rate $\left(R_{1}\right)$, transverse relaxation rate $\left(R_{2}\right)$, and proton-density (PD) have resulted in sequences that can simultaneously measure these physical properties in a reasonable scan time. ${ }^{4-8}$ The advantage of MR imaging quantification is that $\mathrm{R}_{1}, \mathrm{R}_{2}$, and $\mathrm{PD}$ are measured on an absolute scale and are independent of MR imaging scanner settings and imperfections. The multiple parameters can be plotted as a parametric space, where each tissue type has a characteristic range of coordinates. ${ }^{9-11}$ The uptake of GBCA, however, selectively increases the $\mathrm{R}_{1}$ (or, equivalently, decreases the longitudinal $\mathrm{T} 1$ relaxation time, where $\mathrm{T} 1=$ $1 / R_{1}$ ) in comparison with unenhanced tissue, without affecting the $\mathrm{PD}$. Therefore, it is possible to estimate the relative increase in $\mathrm{R}_{1}$ relaxation in a post-GBCA acquisition by taking its $P D$ values and calculating the expected native $R_{1}$ values on the basis of the predetermined relation of native $\mathrm{R}_{1}-\mathrm{PD}$ coordinates of gray matter and white matter. Subtraction of the measured $R_{1}$ values and the calculated native $R_{1}$ values provides an estimate of the $R_{1}$ enhancement map without actually acquiring the native $R_{1}$ values. This procedure can synthesize an absolute $\mathrm{R}_{1}$ enhancement map solely from the post-GBCA acquisition.

The purpose of this study was to create synthetic contrastenhancement images based on a post-GBCA acquisition only and to correlate these with subtraction contrast-enhancement maps in patients with high-grade gliomas. An ROI assessment was performed to get an indication of the sensitivity of the approach.

\section{MATERIALS AND METHODS \\ Subjects}

For this prospective study, 14 patients with typical radiologic images of high-grade gliomas were included. Patient details are provided in Table 1 . The mean age was $64 \pm 10$ years. Patients were examined at baseline before the operations and oncologic treatment. The diagnosis was confirmed after surgery with histopathologic analysis. The regional ethics review board approved the study under approval number 2011/406-31. Written informed consent was obtained from all patients.

\section{MR Imaging Quantification Sequence}

The multiecho, multidynamic sequence to retrieve $\mathrm{R}_{1}, \mathrm{R}_{2}$, and $\mathrm{PD}$ maps has been described previously. ${ }^{7}$ Acquisition details are the following: $\mathrm{FOV}=220 \times 180,24$ slices, voxel size $=0.43 \times 0.43 \times$
$5 \mathrm{~mm}$ (gap $=1 \mathrm{~mm})$. Eight images per slice were measured with $\mathrm{TE}=22$ or $95 \mathrm{~ms}$; and $\mathrm{TI}=170,670,1840$, or $3840 \mathrm{~ms}$ at a TR of $4000 \mathrm{~ms}$. The MR imaging scanner was a $7503 \mathrm{~T}$ system (GE Healthcare, Milwaukee, Wisconsin, with Magnetic Resonance Image Compilation), using the 32-channel head coil. The scan time was 5 minutes and 55 seconds. The sequence was obtained both before and after GBCA injection, with a delay time after injection of about 10 minutes.

\section{$R_{1}$ Enhancement Calculation}

As shown by West et al, ${ }^{12}$ the mean position of native cortical gray matter is at $R_{1}=0.77$ seconds $^{-1}$ with $\mathrm{PD}$ at $86.88 \%$ and the mean position of native white matter is at $\mathrm{R}_{1}=1.38$ seconds $^{-1}$ with $\mathrm{PD}$ at $65.60 \%$ at $3 \mathrm{~T}$. All partial volumes of GM and WM between the mean positions are approximately linear. If one assumes a linear relationship, every $1 \%$ change of the $\mathrm{PD}$ value is associated with a 0.029 second $^{-1}$ change in the $R_{1}$ value. All measured $R_{1}$ and $P D$ combinations in the post-GBCA acquisition were projected onto the line between the gray matter and white matter coordinates. $\mathrm{R}_{1}$ enhancement was then calculated as the measured $R_{1}$ value minus the estimated native $R_{1}$ value. A minimum threshold of $0.2 \mathrm{sec}-$ onds ${ }^{-1}$ was applied to suppress $95 \%$ of the noisy background.

For comparison, subtraction $\mathrm{R}_{1}$ enhancement maps were calculated by performing manual coregistration of the native $R_{1}$ maps using in-plane transformation (rotation and translation) and subtracting the native $R_{1}$ maps from the post-GBCA $R_{1}$ maps.

\section{Synthetic T1-Weighted Images}

Synthetic T1-weighted images were reconstructed on the basis of the measured $\mathrm{R}_{1}, \mathrm{R}_{2}$, and PD maps. The expected signal strength, $S$, in a synthetic T1-weighted image is calculated according to $S=$ $\mathrm{PD} \times\left[1-\exp \left(-\mathrm{R}_{1} \times \mathrm{TR}\right] \times \exp \left(-\mathrm{R}_{2} \times \mathrm{TE}\right)\right.$. The TE was set to 10 $\mathrm{ms}$; the TR was set to $500 \mathrm{~ms}$. Calculation and visualization of the quantitative maps and synthetic T1-weighted images were performed with SyMRI 8.0 (SyntheticMR, Linköping, Sweden).

\section{ROI Placement}

Synthetic T1-weighted images were transferred to the software MeVisLab 2.7 (MeVis Medical Solutions, Bremen, Germany), and ROIs were drawn by 1 neuroradiologist (I.B.), blinded to the clinical information, on the synthetic post-GBCA T1-weighted images to delineate the contrast-enhancing part of the tumor. Care was taken to include the entire enhancing part of the tumor inside the drawn ROI line.

\section{Statistical Analysis}

Linear regression analysis was used to estimate the slope and intercept of the synthetic $R_{1}$ enhancement maps as a function of the subtraction $\mathrm{R}_{1}$ enhancement maps for each subject. The Pearson correlation coefficient was applied to estimate the correlation per subject. The coefficient of variation was calculated as the SD of the difference in synthetic and subtraction $\mathrm{R}_{1}$ enhancement maps divided by the mean of the post-GBCA $\mathrm{R}_{1}$ map, also per subject. For all analyses, only voxels that had a $R_{1}$ enhancement $\left(\mathrm{dR}_{1}\right)$ of $>0.2$ seconds ${ }^{-1}$ were included to avoid a large number of voxels at $(0,0)$, which would bias the intercept. No measures were obtained to suppress residual coregistration artifacts in the subtraction $\mathrm{R}_{1}$ 

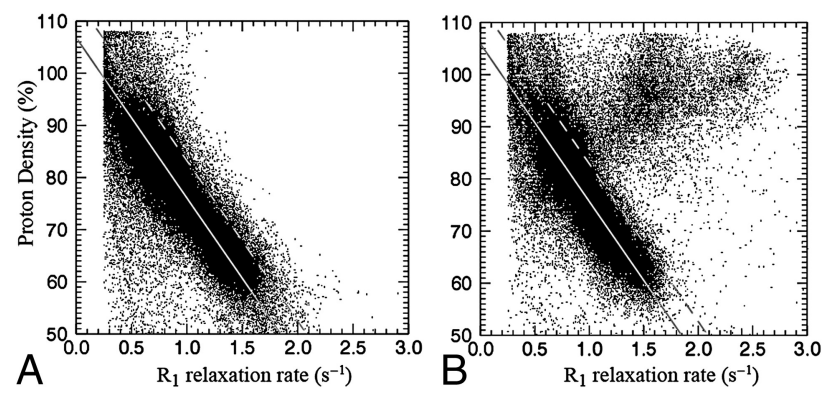

FIG 1. A, Measured proton-density values as a function of $R_{1}$ relaxation rate values of a slice of a brain of a patient with glioma grade IV before administration of GBCA (at 3T). The solid line traverses the average position of gray matter and white matter, indicating the predetermined, linear relationship between $R_{1}$ and PD for the native brain parenchyma. The dotted line indicates a threshold of 0.2 seconds $^{-1}$ from the solid line. $B, P D$ and $R_{1}$ of the same slice after GBCA administration in which the present glioma exhibits enhancement. Some $R_{1}$ values are substantially increased above the dotted threshold line. The estimated $R_{1}$ enhancement corresponds to the measured $R_{1}$ value minus the corresponding $R_{1}$ value on the predetermined solid line.

enhancement maps. The mean and SD for all subjects were calculated using all individual slopes, intercepts, Pearson correlation coefficients, and coefficients of variation. For the ROI analysis, all pixels in the images touched by the ROI lines were selectively analyzed for $\mathrm{R}_{1}$ enhancement using mean and SD. The ROI lines were expanded by 1 and $2 \mathrm{~mm}$ using regiongrowing to make an analysis on an ROI volume with a larger margin around the tumor.

\section{RESULTS}

The parametric representation of $\mathrm{R}_{1}$ enhancement due to GBCA is illustrated in Fig 1. In Fig $1 A$, the $\mathrm{R}_{1}$ and PD values of a slice of a brain are plotted before GBCA administration. It can be clearly seen that the relation between $\mathrm{R}_{1}$ and $\mathrm{PD}$ for the entire brain parenchyma is approximately linear, as indicated by the gray line. In Fig $1 B$, the same slice is depicted after GBCA administration. The $R_{1}$ values within an enhancing part of the tumor have shifted to much higher values, substantially beyond the normal, native $\mathrm{R}_{1}-\mathrm{PD}$ combinations. The rest of the brain remains largely unchanged. The synthetic $R_{1}$ enhancement map is calculated using the difference of post-GBCA $\mathrm{R}_{1}$ values and the estimated native $\mathrm{R}_{1}$ values on the predetermined line. In Fig 2, the same slice of the brain is shown. Synthetic T1-weighted images ( $A$ and $B$ ) are generated using the $\mathrm{R}_{1}, \mathrm{R}_{2}$, and $\mathrm{PD}$ maps of the quantification sequence. The enhancement due to administration of GBCA is clearly visible on the T1-weighted images, as well as on the $R_{1}$ maps $(C$ and $D)$. The native $\mathrm{R}_{1}$ maps are coregistered to the postGBCA $\mathrm{R}_{1}$ maps to obtain the subtraction $\mathrm{R}_{1}$ enhancement $(E)$. High-intensity enhancement corresponds to a $\mathrm{dR}_{1}$ in the range of $1.5-2.5$ seconds $^{-1}$. The diffuse signals throughout the entire volume are due to imperfect image coregistration of the anatomic details. In Fig $2 F$, the synthetic $\mathrm{R}_{1}$ enhancement map, based on the post-GBCA acquisition only, is shown. Linear regression of the subtraction and synthetic $\mathrm{R}_{1}$ enhancement maps on all patients showed a mean slope of $1.02 \pm 0.19$ and mean intercept of $0.05 \pm$ 0.12 . Statistically, the unity line at intercept zero could not be ruled out. The mean Pearson correlation coefficient of all patients was $0.861 \pm 0.059$. The mean coefficient of variation of

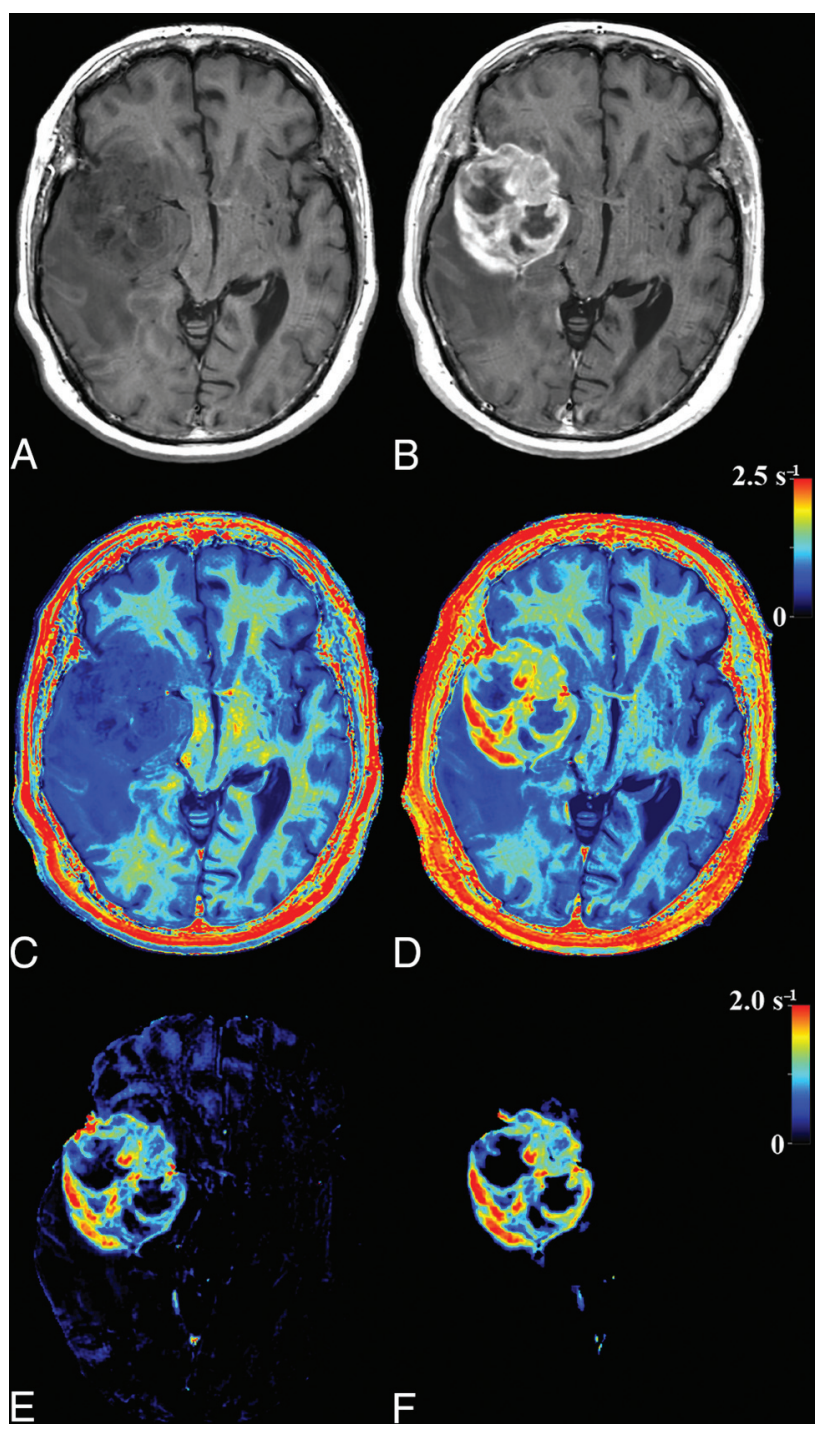

FIG 2. Images of the same slice as in Fig 1: synthetic T1-weighted imaging using native data $(A)$, synthetic T1-weighted imaging using post-GBCA data $(B)$, the native $R_{1}$ map $(C)$, the post-GBCA $R_{1}$ map $(D)$, the difference map of the coregistered native map $(E)$, and the postGBCA $R_{1}$ synthetic-difference map based on the post-GBCA acquisition only $(F)$.

all patients was $0.18 \pm 0.04$. In Fig 3, a 2D histogram is plotted of the detected $\mathrm{R}_{1}$ enhancement using subtraction of the native and post-GBCA $R_{1}$ maps as a function of synthetic $R_{1}$ maps for all included patients.

In Fig 4, the tumor in Fig 2 is zoomed-in. The native and post-GBCA T1-weighted images are shown as well as the ROI drawn by the radiologist. In Fig $4 D$, the synthetic $\mathrm{R}_{1}$ enhancement map is shown as a green overlay where full color corresponds to a $\mathrm{dR}_{1}$ of 1 second $^{-1}$. At various places, low-intensity enhancement in the range $0.2-0.5$ seconds $^{-1}$ is observable outside the highintensity enhancing tumor and drawn ROI. On average for all patients, $35.8 \%$ of the pixels touched by the drawn ROI lines had values above 0.2 seconds ${ }^{-1}$ for the synthetic $R_{1}$ enhancement map and even $50.3 \%$ for the subtraction $\mathrm{R}_{1}$ enhancement map. When the ROI line was expanded with an additional margin of 1 or 2 $\mathrm{mm}$, this percentage reduced to $8.0 / 17.4 \%$ and $2.3 / 8.6 \%$, respectively (Table 2). 
In 10 of 14 patients, $>1 \mathrm{~mL}$ of tissue was found in the synthetic $\mathrm{R}_{1}$ enhancement images with an enhancement of $>0.2$ seconds $^{-1}$ outside the drawn ROI. The mean additional tumor volume for all patients was $1.71 \pm 1.28 \mathrm{~mL}$, with a maximum of $4.3 \mathrm{~mL}$. In comparison, the mean tumor volume within the ROIs was $63.5 \pm$ $44.4 \mathrm{~mL}$ (range, 9-134 mL). More examples of the synthetic $\mathrm{R}_{1^{-}}$ enhancement maps are provided in Fig 5.

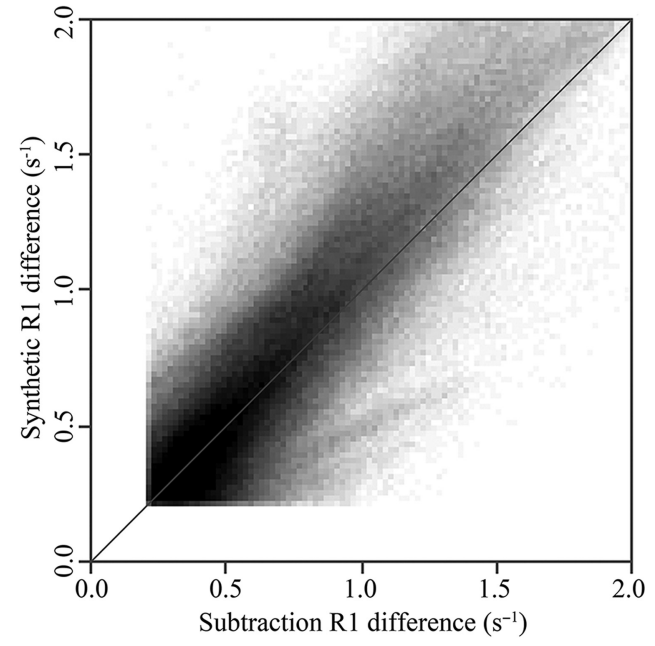

FIG 3. $2 D$ histogram of the $R_{1}$ enhancement found using the subtraction of native and post-GBCA $R_{1}$ maps as a function of the synthetic $R_{1}$ enhancement, based on the post-GBCA acquisition only, of all included patients. The black and white intensity in the plot is proportional to the number of times an $x, y$ coordinate occurred. The diagonal line indicates equivalence.

\section{DISCUSSION}

Detection of GBCA enhancement is an important clinical biomarker, which generally is used in a qualitative manner because a native T1-weighted image is subjectively compared with the postGBCA T1-weighted image by the radiologist. Fortunately, quantitative MR imaging, including measurement of the absolute $\mathrm{R}_{1}$ relaxation rates, is increasingly available and clinically supported. ${ }^{13}$ Some reports exist on the application of quantitative MR imaging to gliomas. ${ }^{14-16}$ The use of $\mathrm{R}_{1}$ maps is expected to be more sensitive than conventional T1-weighted imaging. As observed in Fig 1, the increase of the $\mathrm{R}_{1}$ of enhancing tumors is on the order of 2 seconds $^{-1}$, starting at a native value of about 0.5 seconds ${ }^{-1}$. This corresponds to a relative increase of $400 \%$. A conventional T1-weighted TSE image, on the other hand, is an exponentially saturated image, proportional to $1-\exp \left(-\mathrm{R}_{1} \times\right.$ TR). The relative increase of signal strength in a T1-weighted image, using the same $\mathrm{R}_{1}$ values and a TR of $500 \mathrm{~ms}$, is only $220 \%$. The true advantage of $\mathrm{R}_{1}$ mapping, however, is that these maps are not obscured by PD, $\mathrm{B}_{1}$, and coil-sensitivity differences.

Accurately measuring the quantitative $\mathrm{R}_{1}$ enhancement due to GBCA administration in clinical practice, however, is still challenging due to patient motion. Patients tend to change position between acquisitions, and image subtraction requires robust, enhancement-independent image coregistration. ${ }^{17}$ Especially, subtle, low-intensity enhancement areas at the edges of a high-intensity tumor are easily corrupted by residual anatomic detail (Fig $2 E$ ). The lack of confidence in such areas generally results in ignoring them, which can have an impact on treatment and outcome. ${ }^{18,19}$ Our study shows that quantitative $\mathrm{R}_{1}$ enhancement can be found using the post-GBCA acquisition only, removing the

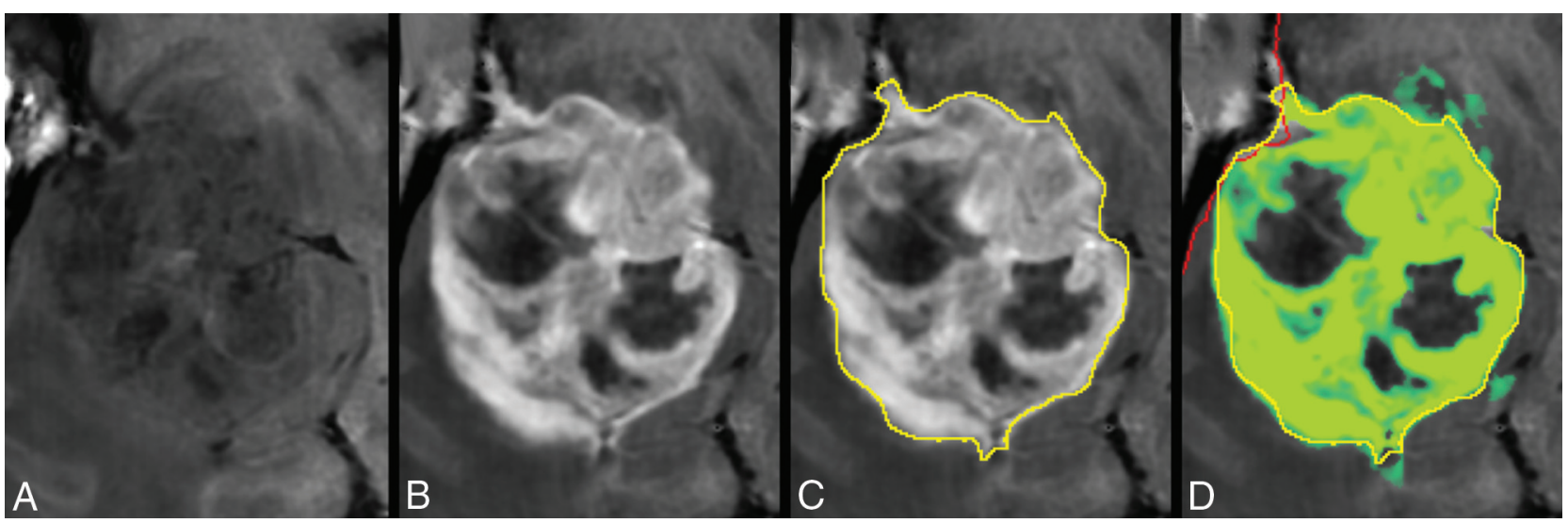

FIG 4. Zoomed part around the tumor displayed in Fig 2. Synthetic T1-weighted imaging using native data (A), synthetic T1-weighted imaging using post-GBCA data $(B)$, the $R O I$ line as drawn by a neuroradiologist to encapsulate the border of the enhancing tumor $(C)$. $D$, Synthetic $R_{1}$ enhancement map shown as a green overlay on the synthetic Tl-weighted image in which full color corresponds to $\mathrm{dR}_{1}=1.0$ seconds $^{-1}$. The minimum enhancement was set at $\mathrm{dR}_{1}=0.2$ seconds $^{-1}$. Some low-intensity enhancement is visible outside the yellow ROI. The red line indicates the edge of the intracranial volume.

Table 2: Observed $R_{1}$ enhancement of the pixels of the ROI line drawn by a neuroradiologist to encapsulate the enhancing tumor, as a percentage of all values above $\mathrm{dR}_{1}=0.2$ seconds $^{-1}$, the mean $\mathrm{dR}_{1}$, and the mean $\mathrm{dR}_{1}$ of all values of $>0.2$ seconds $^{-1 a}$

\begin{tabular}{|c|c|c|c|c|c|c|}
\hline & \multicolumn{2}{|c|}{$\mathrm{dR}_{1}>0.2 \mathrm{~s}^{-1}(\%)$} & \multicolumn{2}{|c|}{ Mean $\mathrm{dR}_{1}\left(\mathrm{~s}^{-1}\right)$} & \multicolumn{2}{|c|}{ Mean $\mathrm{dR}_{1}\left(>0.2 \mathrm{~s}^{-1}\right.$ only) $\left(\mathrm{s}^{-1}\right)$} \\
\hline & Synthetic & Subtraction & Synthetic & Subtraction & Synthetic & Subtraction \\
\hline On ROI line & $35.8 \pm 14.3$ & $50.3 \pm 10.2$ & $0.19 \pm 0.09$ & $0.27 \pm 0.08$ & $0.48 \pm 0.12$ & $0.46 \pm 0.11$ \\
\hline$+1 \mathrm{~mm}$ & $8.0 \pm 5.8$ & $17.4 \pm 9.0$ & $0.03 \pm 0.02$ & $0.08 \pm 0.05$ & $0.35 \pm 0.08$ & $0.37 \pm 0.12$ \\
\hline$+2 \mathrm{~mm}$ & $2.3 \pm 1.5$ & $8.6 \pm 4.9$ & $0.01 \pm 0.01$ & $0.04 \pm 0.04$ & $0.32 \pm 0.15$ & $0.37 \pm 0.17$ \\
\hline
\end{tabular}

${ }^{a}$ Results are listed for the $R_{1}$ difference generated by synthesizing the $R_{1}$ difference map and subtraction of the pre- and post-GBCA $R_{1}$ maps. Two more ROI lines were created at 1- and 2-mm outward to analyze the results if a larger margin around the enhancing tumor had been drawn. 


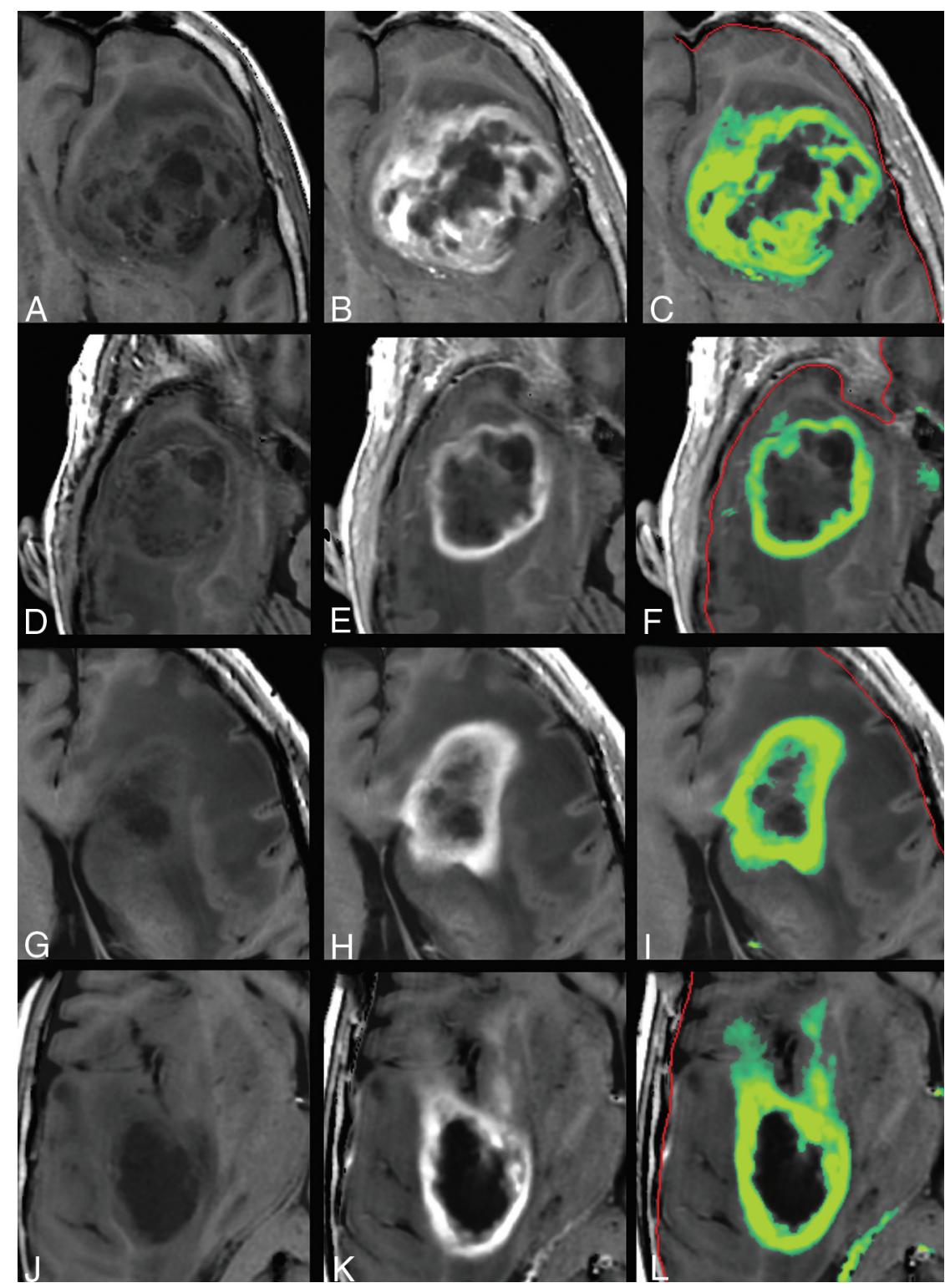

FIG 5. Other examples of the synthetic $R_{1}$ enhancement map and low-intensity enhancement at the edges of high-intensity enhancement in gliomas. Left: native synthetic T1-weighted image. Center: post-GBCA synthetic T1-weighted image. Right: synthetic $\mathrm{R}_{1}$ enhancement map as a green overlay. The color indicates a range of $\mathrm{dR}_{1}$ of $0.2-1.0$ seconds $^{-1}$. The red line indicates the edge of the intracranial volume.

image coregistration issue entirely. Linear regression between our proposed method and image subtraction was observed to be very close to unity (slope of 1.02, intercept of 0.05 ). This opens the opportunity to objectively measure $\mathrm{R}_{1}$ enhancement without the acquisition of the native $R_{1}$ maps. The removal of image coregistration issues may improve the sensitivity of low-enhancement areas and allow an objective threshold for tumor delineation.

To illustrate the perception of a radiologist, we drew ROIs to encapsulate the enhancing part of the tumor. For our 14 subjects, $36 \%$ of the pixels touched by the ROI line had $\mathrm{dR}_{1}$ values above the chosen threshold of 0.2 seconds $^{-1}$, with a mean enhancement of 0.48 seconds $^{-1}$. For the subtraction $\mathrm{R}_{1}$-enhancement map, it was $50 \%$, with similar mean enhancement. This indicates that the perception of the trained eye to determine $\mathrm{R}_{1}$ enhancement is on the order of $0.2-0.5$ seconds $^{-1}$, corresponding to $10 \%-25 \%$ of the maximum enhancement. Further studies, with more readers are required to verify this value, but it shows that lowintensity enhancement in a T1-weighted image is easily rated as nonenhancing, which can affect the diagnosis. ${ }^{20,21}$ It is well-known that high-grade gliomas infiltrate into the peritumoral edema, ${ }^{3,16}$ which can be detected with higher sensitivity using diffusion ${ }^{22}$ or a multiparametric approach. ${ }^{23}$ In our study, the application of an additional peritumoral margin of 1 or $2 \mathrm{~mm}$ rapidly reduced the number of pixels above the threshold as well as the mean $\mathrm{dR}_{1}$ on the ROI line. For those pixels that did have values above 0.2 seconds ${ }^{-1}$, however, the mean $\mathrm{dR}_{1}$ was $0.3-0.4$ seconds $^{-1}$, indicating that the enhancement was highly localized. Examples are shown in Figs 4 and 5. Low-intensity enhancement at the tumor edges is not distributed equally on all sides; it occurs mainly in a limited number of focal areas. In a previous study, we showed a gradient of $\mathrm{R}_{1}$ relaxation at the edge of enhancing tumors. ${ }^{16}$ The current study indicates that the detected gradient was likely to be a composition of no-gradient areas and high-gradient areas.

A limitation of this study is the small number of patients and the use of a specific pathology. It can be speculated that synthetic $R_{1}$ enhancement maps can be generated for all cases of GBCA infiltration, but general use is yet to be confirmed. Furthermore, synthetic $\mathrm{R}_{1}$ enhancement may have other causes than the presence of GBCA, for example, a hematoma or fatty tissue. A larger study would be required to assess the potential implications on diagnostic confidence in the neuroradiology assessment. Secondary reactions due to radiation therapy treatment, which may mimic tumor growth, ${ }^{24}$ were not investigated. No biopsy data were available to confirm a relation with the synthetic low-intensity $\mathrm{R}_{1}$ enhancement and actual tumor infiltration. Larger clinical studies are required to validate our approach and assess the impact of its potential use.

A technical limitation was our assumption of a fixed $\mathrm{R}_{1}-\mathrm{PD}$ relation for the entire brain. This may seem rather coarse, but our study showed that it worked remarkably well. The reason is that the relative increase of $R_{1}$ due to GBCA administration is so large, nearly an order of magnitude larger than the normal variation within brain tissue from the $\mathrm{R}_{1}-\mathrm{PD}$ line. These large GBCA enhancements are typical for clinical routine because the contrast difference in conventional T1-weighted images is not linear and is relatively weak compared with an $\mathrm{R}_{1}$ map. Possibly, quantitative 
MR imaging may therefore permit using lower doses of GBCA. Our proposed synthetic $\mathrm{R}_{1}$ enhancement approach may, in some cases, allow omitting the native T1-weighted images, which would result in a considerable examination time gain. Even if omission proves impossible, the quantitative enhancement measurement may still provide a more objective and sensitive input for drawing the margin around gliomas, especially considering the low-intensity enhancement areas.

\section{CONCLUSIONS}

Our study shows that it is possible to synthesize an $\mathrm{R}_{1}$-enhancement map in patients with high-grade gliomas on the basis of a post-GBCA MR imaging quantification sequence only. A good correlation with subtraction $\mathrm{R}_{1}$-enhancement maps was found. The method may improve the sensitivity and objectivity for enhancement detection, especially for areas with low-intensity enhancement.

Disclosures: Marcel Warntjes-UNRELATED: Employment: SyntheticMR AB, Comments: part-time employment at SyntheticMR AB; Stock/Stock Options: SyntheticMR AB.

\section{REFERENCES}

1. Ellingson BM, Bendszus M, Sorensen AG, et al. Emerging techniques and technologies in brain tumor imaging. Neuro Oncol 2014; 16(Suppl 7):vii12-23 CrossRef Medline

2. Ellingson BM, Harris RJ, Woodworth KL. Baseline pretreatment contrast enhancing tumor volume including central necrosis is a prognostic factor in recurrent glioblastoma: evidence from single- and multicenter trials. Neuro Oncol 2017;19:89-98 CrossRef Medline

3. Claes A, Idema AJ, Wesseling P. Diffuse glioma growth: a guerilla war. Acta Neuropathol 2007;114:443-58 CrossRef Medline

4. Zhu DC, Penn RD. Full-brain T1 mapping through inversion recovery fast spin echo imaging with time-efficient slice ordering. Magn Reson Med 2005;54:725-31 CrossRef Medline

5. Neeb H, Zilles K, Shah NJ. A new method for fast quantitative mapping of absolute water content in vivo. Neuroimage 2006;31:1156-68 CrossRef Medline

6. Deoni SC, Peters TM, Rutt BK. High-resolution T1 and T2 mapping of the brain in a clinically acceptable time with DESPOT1 and DESPOT2. Magn Reson Med 2005;53:237-41 CrossRef Medline

7. Warntjes JB, Leinhard OD, West J, et al. Rapid magnetic resonance quantification on the brain: optimization for clinical usage. Magn Reson Med 2008;60:320-29 CrossRef Medline

8. Ma D, Gulani V, Seiberlich N, et al. Magnetic resonance fingerprinting. Nature 2013;495:187-92 CrossRef Medline

9. Alfano B, Brunetti A, Arpaia M, et al. Multiparametric display of spin-echo data from MR studies of brain. J Magn Reson Imaging 1995;5:217-25 CrossRef Medline

10. Warntjes JB, Engström M, Tisell A, et al. Brain characterization us- ing normalized quantitative magnetic resonance imaging. PLoS One 2013;8:e70864 CrossRef Medline

11. Engström M, Warntjes JB, Tisell A, et al. Multi-parametric representation of voxel-based quantitative magnetic resonance imaging. PLoS One 2014;9:e111688 CrossRef Medline

12. West J, Blystad I, Engström M, et al. Application of quantitative MRI for brain tissue segmentation at $1.5 \mathrm{~T}$ and $3.0 \mathrm{~T}$ field strengths. PLoS One 2013;8:e74795 CrossRef Medline

13. Tanenbaum LN, Tsiouris AJ, Johnson AN, et al. Synthetic MRI for clinical neuroimaging: results of the Magnetic Resonance Image Compilation (MAGiC) prospective, multicenter, multireader trial. AJNR Am J Neuroradiol 2017;38:1103-10 CrossRef Medline

14. Müller A, Jurcoane A, Kebir S, et al. Quantitative T1-mapping detects cloudy-enhancing tumor compartments predicting outcome of patients with glioblastoma. Cancer Med 2017;6:89-99 CrossRef Medline

15. Lescher S, Jurcoane A, Veit A, et al. Quantitative T1 and T2 mapping in recurrent glioblastomas under bevacizumab: earlier detection of tumor progression compared to conventional MRI. Neuroradiology 2015;57:11-20 CrossRef Medline

16. Blystad I, Warntjes JBM, Smedby Ö, et al. Quantitative MRI for analysis of peritumoral edema in malignant gliomas. PLoS One 2017;12: e0177135 CrossRef Medline

17. Ellingson BM, Kim HJ, Woodworth DC, et al. Recurrent glioblastoma treated with bevacizumab: contrast-enhanced T1-weighted subtraction maps improve tumor delineation and aid prediction of survival in a multicenter clinical trial. Radiology 2014;271:200-10 CrossRef Medline

18. Grabowski MM, Recinos PF, Nowacki AS, et al. Residual tumor volume versus extent of resection: predictors of survival after surgery for glioblastoma. J Neurosurg 2014;121:1115-23 CrossRef Medline

19. Chaichana KL, Jusue-Torres I, Navarro-Ramirez R, et al. Establishing percent resection and residual volume thresholds affecting survival and recurrence for patients with newly diagnosed intracranial glioblastoma. Neuro Oncol 2014;16:113-22 CrossRef Medline

20. Eisele SC, Wen PY, Lee EQ. Assessment of brain tumor response: RANO and its offspring. Curr Treat Options Oncol 2016;17:35 CrossRef Medline Medline

21. Shiroishi MS, Boxerman JL, Pope WB. Physiologic MRI for assessment of response to therapy and prognosis in glioblastoma. Neuro Oncol 2016;18:467-78 CrossRef Medline

22. Lemercier P, Paz Maya S, Patrie JT, et al. Gradient of apparent diffusion coefficient values in peritumoral edema helps in differentiation of glioblastoma from solitary metastatic lesions. AJR Am J Roentgenol 2014;203:163-69 CrossRef Medline

23. Akbari H, Macyszyn L, Da X, et al. Imaging surrogates of infiltration obtained via multiparametric imaging pattern analysis predict subsequent location of recurrence of glioblastoma. Neurosurgery 2016; 78:572-80 CrossRef Medline

24. Hygino da Cruz LC Jr, Rodriguez I, Domingues RC, et al. Pseudoprogression and pseudoresponse: imaging challenges in the assessment of posttreatment glioma. AJNR Am J Neuroradiol 2011;32: 1978-85 CrossRef Medline 